\title{
Time Delay Estimation for KSP-OFDM Systems in Multipath Fading Channels
}

\author{
Dieter Van Welden, Heidi Steendam and Marc Moeneclaey \\ TELIN Department, Ghent University \\ Sint-Pietersnieuwstraat 41, 9000 Gent, Belgium \\ E-mail: $\{$ dmvwelde,hs,mm $\} @$ telin.ugent.be
}

\begin{abstract}
In this paper we present a time delay estimation algorithm for known symbol padding (KSP) orthogonal frequency division multiplexing (OFDM) systems. The estimator exploits both the pilot symbols in the guard interval and on the pilot carriers, and takes the frequency selectivity of the fading channel into account. Our simulations indicate that the time delay estimator gives rise to only a small degradation of the bit error rate (BER) performance, as compared to a receiver with perfect synchronization.
\end{abstract}

\section{INTRODUCTION}

Orthogonal frequency division multiplexing (OFDM) has proven to be an interesting technique to achieve high data rates and to cope with multipath fading channels [1]. In order to avoid inter block interference (IBI) between adjacent OFDM blocks, an OFDM system makes use of a guard interval between consecutive OFDM blocks. Different types of guard interval, have been proposed in literature. The most popular are the cyclic prefix $(\mathrm{CP})$ and the zero padding $(\mathrm{ZP})$ techniques [2]. In the $\mathrm{CP}$ technique, the guard interval is transmitted before each OFDM block and is filled with the last samples of the OFDM block. In ZP-OFDM, the guard interval consists of zeros, i.e. during the guard interval no signal is transmitted. Recently a third technique has been proposed, i.e. the known symbol padding (KSP) technique [3], [4]. In this technique, the guard interval is filled with known samples, that are referred to as time-domain pilots. Here we will focus on KSP-OFDM.

An important issue with OFDM systems is their much higher sensitivity to some synchronization errors, as compared to single carrier systems [5], so synchronizing with the transmitter is an important task of the OFDM receiver. One aspect of the synchronization process comprises finding the starting point of the OFDM symbol; accurate time delay estimation is necessary because time offsets can cause inter carrier interference (ICI) and IBI [6], [7]. Several time delay estimation algorithms for CP-OFDM have already been proposed in the literature. In [8], the maximum likelihood (ML) estimator for a time delay in the presence of additive white Gaussian noise (AWGN) has been presented. The algorithm exploits the redundancy of the cyclic prefix and the pilot symbols on the carriers. In [9], the authors propose an estimator which makes use of a specially designed training symbol for the AWGN channel. However in multipath fading environments, the mentioned estimators result in inaccurate estimates with large biases. To guarantee a good performance, the OFDM system would require a long guard interval, which reduces efficiency in terms of both power and bandwidth. In literature, there are some contributions which take the frequency selective nature of the channel in to account. The authors of [10] derive the ML time delay estimator in the case of a dispersive channel under the assumption of perfect channel knowledge. The algorithm exploits the cyclic prefix only. However, in practice this estimator is not applicable because it is very difficult to obtain a channel estimate without knowledge about the time delay. This estimator can only serve as a lower bound on the performance of an estimator which does not assume any knowledge about the channel. In [11], a coarse time delay estimate is obtained by applying the correlation method based on the cyclic prefix. The coarse estimate is further improved by employing a path time delay estimation method. The algorithm can show a good performance, but it is not that easy to choose an appropriate value for the correction term of the coarse estimate and for the threshold value for the path time delay estimation.

It has been shown in [4] that, under perfect synchronization, CP-OFDM and KSP-OFDM have essentially the same BER performance when the number of guard interval samples is much smaller than the number of carriers. As in all practical situations, one wants to keep the necessary overhead as small as possible, we were motivated to consider the timing synchronization problem for KSP-OFDM, where both time-domain pilots (in the guard interval) and frequency-domain pilots (on some of the carriers) are used. To our knowledge, no research has been done about algorithms for the timing synchronization problem for KSP-OFDM. Our estimator exploits both the pilot symbols in the guard interval and the pilot symbols on the pilot carriers. The proposed algorithm is compared to the estimator for CP-OFDM from [11] in terms of their effect on the bit error rate (BER) performance.

\section{SYSTEM MODEL}

We consider a KSP-OFDM system that consists of $N$ carriers. The different carriers are filled with blocks of symbols, $\mathbf{a}_{i}=\left(a_{i}(0), \ldots, a_{i}(N-1)\right)^{T}$, where $i$ denotes the block index and $a_{i}(n)$ is the symbol on the carrier with index $n(n=0, \ldots, N-1)$ during the $i$-th block. The symbol vector $\mathbf{a}_{i}$ is applied to an $N$ point inverse fast Fourier transform (IFFT) to modulate the symbols on the different carriers. The resulting $N$ IFFT outputs constitute the first 
a) transmitter

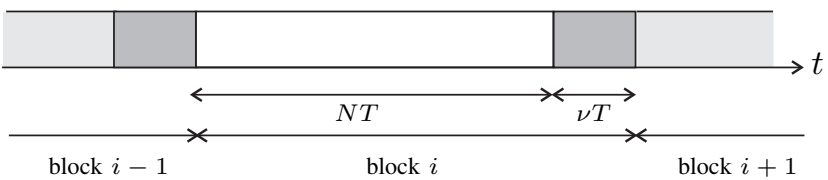

b) receiver

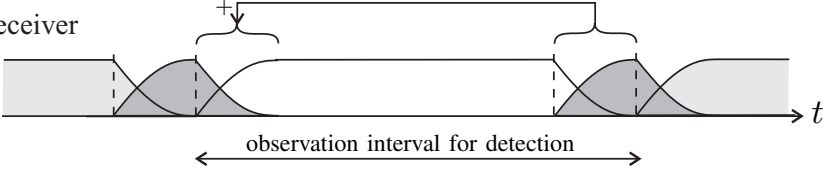

Fig. 1. Time-domain signal of a KSP-OFDM block a) transmitted signal b) received signal and observation interval for detection

$N$ time-domain samples of the $i$-th OFDM block. In order to avoid IBI between the symbols from consecutive OFDM blocks, a guard interval of $\nu$ time-domain samples is appended to each OFDM block. The resulting $N+\nu$ samples $\mathbf{s}_{i}=$ $\left(s_{i}(0), \ldots, s_{i}(N+\nu-1)\right)^{T}$ of the $i$-th OFDM block are given by

$$
\mathbf{s}_{i}=\sqrt{\frac{N}{N+\nu}}\left(\begin{array}{c}
\mathbf{F}^{H} \mathbf{a}_{i} \\
\mathbf{b}_{g}
\end{array}\right)
$$

where $\mathbf{F}$ denotes the $N \times N$ FFT matrix with elements $(\mathbf{F})_{k, l}=\frac{1}{\sqrt{N}} e^{-j 2 \pi \frac{k l}{N}} ; k, l=0, \ldots, N-1$, and $\mathbf{b}_{g}=$ $\left(b_{g}(0), \ldots, b_{g}(\nu-1)\right)^{T}$ is the vector of $\nu$ guard interval samples. Furthermore, $M-\nu$ carriers are filled with pilot symbols, so that a number of $M$ pilot symbols are transmitted per OFDM block. The symbols on the pilot carriers are denoted as $\mathbf{b}_{c}=\left(b_{c}(0), \ldots, b_{c}(M-\nu-1)\right)^{T}$. The remaining $N-$ $M+\nu$ carriers are filled with data symbols and are defined as $\mathbf{a}_{d}^{(i)}=\left(a_{d}^{(i)}(0), \ldots, a_{d}^{(i)}(N-M+\nu-1)\right)^{T} . E_{s}$ denotes the transmitted energy per symbol: $E_{s}=\mathrm{E}\left[\left|a_{i}(n)\right|^{2}\right]=$ $\mathrm{E}\left[\left|b_{g}(k)\right|^{2}\right]$. The time domain signals at the transmitter and at the receiver side are shown in figure 1.

We define the vectors $\mathbf{s}_{p}$ and $\mathbf{s}_{d}^{(i)}$ as the time domain signal samples corresponding to the pilot carriers and the data carriers respectively:

$$
\begin{aligned}
\mathbf{s}_{p} & =\sqrt{\frac{N}{N+\nu}} \mathbf{F}_{p} \mathbf{b}_{c} \\
\mathbf{s}_{d}^{(i)} & =\sqrt{\frac{N}{N+\nu}} \mathbf{F}_{d} \mathbf{a}_{d}^{(i)}
\end{aligned}
$$

where $\mathbf{F}_{p}$ is given by the $M-\nu$ columns of $\mathbf{F}^{H}$ which correspond to the pilot carriers and $\mathbf{F}_{d}$ consists of the remaining $N-M+\nu$ columns of $\mathbf{F}^{H}$ that correspond to the data carriers. The vector $\mathbf{b}$ is defined as the total transmitted pilot signal vector, so $\mathbf{b}$ collects the samples of $\mathbf{s}_{p}$ and the pilot symbols in the guard interval

$$
\mathbf{b}_{i}=\left(\frac{\mathbf{s}_{p}}{\sqrt{\frac{N}{N+\nu}} \mathbf{b}_{g}}\right) .
$$

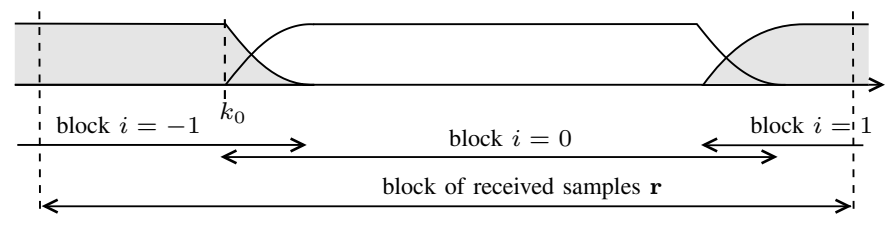

Fig. 2. Definition of the received signal vector $\mathbf{r}$

The components of $\mathbf{s}_{i}$ are transmitted over a frequency selective channel with an impulse response consisting of $L$ taps, denoted as $\mathbf{h}=(h(0), \ldots, h(L-1))^{T}$. In order to avoid IBI, the length of the guard interval is chosen so that the guard interval exceeds the duration of the channel impulse response: $\nu \geq L-1$.

At the receiver, we consider a block of $2(N+\nu)+L-2$ received signal samples $\mathbf{r}=$ $(r(0), \ldots, r(2(N+\nu)+L-3))^{T}$ (see figure 2). Every transmitted OFDM block has a duration of $N+\nu$ samples, so after transmission over a channel with an impulse response of $L$ samples, every transmitted OFDM block will contribute to $N+\nu+L-1$ successive samples of the received signal. Because of its length, the vector $\mathbf{r}$ contains the total contribution from only one OFDM block (along with partial contributions from adjacent blocks). Without loss of generality we can give this block the index $i=0$. The starting point $k_{0}$ of this block in the received signal vector $\mathbf{r}$ is unknown and has to be estimated.

For the data detection of block $i$, we take the $N+\nu$ samples of the received signal from the observation interval corresponding to block $i$ shown in figure 1 . The contributions from the time-domain pilots (dark grey areas on figure 1) are first subtracted from the received signal. The resulting system is in fact a ZP-OFDM system. The data detection in a ZPOFDM system is very similar to data detection in a CP-OFDM system [2]: The last $\nu$ samples of the observation interval are added to the first $\nu$ samples of the OFDM symbol (see figure 1b). The resulting block of $N$ samples is then applied to the FFT. Finally one-tap equalization and symbol detection are performed on each FFT output. In order to subtract the contribution from the time-domain pilots and to perform onetap equalization, accurate estimates of the starting point $k_{0}$ of the observation interval and of the channel impulse response $\mathbf{h}$ must be available. This contribution focuses on the estimation of $k_{0}$.

\section{Time Delay Estimation}

In this section an estimator for $k_{0}$ is derived, starting from the joint likelihood function of $k_{0}$ and $\mathbf{h}$ for the observation $\mathbf{r}$. To simplify the derivation of the estimator, we assume that $\mathbf{r}$ only contains noise besides the contribution of the considered transmitted OFDM block $\mathbf{s}_{0}{ }^{1}$. For notational convenience, the block index $i=0$ is dropped from now on. We define the vector $\mathbf{r}_{0}$ as the subvector of $\mathbf{r}$ that contains the contributions

\footnotetext{
${ }^{1}$ This assumption is only used to derive the estimator, for the simulations we will consider a continuous transmission of consecutive OFDM blocks.
} 
from s: $\mathbf{r}_{0}=\left(r\left(k_{0}\right), \ldots, r\left(k_{0}+N+\nu+L-2\right)\right)^{T}$. Because of the already made assumption, the vector $r_{0}$ can be expressed as

$$
\mathbf{r}_{0}=\mathbf{H s}+\mathbf{w}
$$

where $\mathbf{s}$ is defined in (1) (with $i=0$ ), $\mathbf{w}=$ $\left(w\left(k_{0}\right), \ldots, w\left(k_{0}+N+\nu+L-2\right)\right)^{T}$ is the noise vector and $w(k)$ is additive white Gaussian noise with variance $N_{0}$ and zero mean, and $\mathbf{H}$ is an $(N+\nu+L-1) \times(N+\nu)$ matrix with a Toeplitz structure. The entries of $\mathbf{H}$ are given by $(\mathbf{H})_{l: l+L-1, l}=\mathbf{h} ; l=0, \ldots, L-1$. The useful signal in (5) can be written as the sum of the contribution of the data symbols and the pilot symbols:

$$
\mathbf{H s}=\mathbf{B h}+\mathbf{A h}
$$

where $\mathbf{B}$ and $\mathbf{A}$ are the $(N+\nu+L-1) \times L$ Toeplitz matrices with respective entries $(\mathbf{B})_{l: l+N+\nu-1, l}=\mathbf{b}$ and $(\mathbf{A})_{l: l+N-1, l}=\mathbf{s}_{d} ; l=0, \ldots, L-1$.

The joint probability of the samples of $\mathbf{r}$ given $k_{0}$, the channel impulse response $\mathbf{h}$, and the data symbol vector $\mathbf{a}_{d}$ is given by

$$
\begin{gathered}
p\left(\mathbf{r} \mid k_{0}, \mathbf{h}, \mathbf{a}_{d}\right)= \\
C \exp \left\{-\frac{1}{N_{0}}\left(\sum_{k=0}^{k_{0}-1}|r(k)|^{2}+\sum_{k=k_{0}+N+\nu+L-1}^{2(N+\nu+L-2)}|r(k)|^{2}\right)\right\} . \\
\quad \exp \left\{-\frac{1}{N_{0}}\left[\mathbf{r}_{0}-(\mathbf{B}+\mathbf{A}) \mathbf{h}\right]^{H}\left[\mathbf{r}_{0}-(\mathbf{B}+\mathbf{A}) \mathbf{h}\right]\right\}
\end{gathered}
$$

where $C$ is some irrelevant constant. This expression is still dependent on the unknown data symbols $\mathbf{a}_{d}$. To get rid of this vector, we have two options. A first option is to average (7) over the unknown data symbols. This averaging is rather complicated so (7) has to be simplified first. For small values of $x, \exp (x)$ can be approximated by the first two terms of its Taylor series, i.e. $\exp (x)=1+x$ for $|x| \ll 1$. So for low signal to noise ratios, expression (7) can be approximated by

$$
\begin{aligned}
p\left(\mathbf{r} \mid k_{0}, \mathbf{h}, \mathbf{a}_{d}\right)= & \\
C-\frac{C}{N_{0}} & \left(\sum_{k=0}^{k_{0}-1}|r(k)|^{2}+\sum_{k=k_{0}+N+\nu+L-1}^{2(N+\nu+L-2)}|r(k)|^{2}\right) \\
& -\frac{C}{N_{0}}\left[\mathbf{r}_{0}-(\mathbf{B}+\mathbf{A}) \mathbf{h}\right]^{H}\left[\mathbf{r}_{0}-(\mathbf{B}+\mathbf{A}) \mathbf{h}\right] .
\end{aligned}
$$

The averaging of (8) over the unknown data symbols is easily performed as we only need to compute the averages of $\mathbf{A}$ and $\mathbf{A}^{H} \mathbf{A}: \mathbf{E}[\mathbf{A}]=0$ and $\mathrm{E}\left[\mathbf{A}^{H} \mathbf{A}\right]=\mathbf{R}_{\mathbf{A}}$ (See appendix for more details). This yields for $p\left(\mathbf{r} \mid k_{0}, \mathbf{h}\right)$

$$
\begin{array}{r}
p\left(\mathbf{r} \mid k_{0}, \mathbf{h}, \mathbf{b}_{c}, \mathbf{b}_{g}\right)= \\
C\left\{1-\frac{1}{N_{0}}\left[\mathbf{r}^{H} \mathbf{r}-\mathbf{r}_{0}^{H} \mathbf{B h}-\mathbf{h}^{H} \mathbf{B}^{H} \mathbf{r}_{0}\right]\right. \\
\left.\quad-\frac{1}{N_{0}} \mathbf{h}^{H}\left(\mathbf{B}^{H} \mathbf{B}+\mathbf{R}_{\mathbf{A}}\right) \mathbf{h}\right\} .
\end{array}
$$

The ML estimates of $k_{0}$ and $\mathbf{h}$ are obtained by maximizing the probability function of (9) with respect to $k_{0}$ and $\mathbf{h}$. The estimate $\hat{\mathbf{h}}$ can be expressed as a function of $k_{0}$ and is obtained by equating to zero the derivative of (9) with respect to $\mathbf{h}$. This yields

$$
\hat{\mathbf{h}}\left(k_{0}\right)=\left(\mathbf{B}^{H} \mathbf{B}+\mathbf{R}_{\mathbf{A}}\right)^{-1} \mathbf{B}^{H} \mathbf{r}_{0} .
$$

To find the ML estimate of $k_{0}$, we substitute the estimate of $\mathbf{h}$ in (9). We obtain the function $\Gamma_{1}\left(k_{0}\right)$ which only depends on $k_{0}$ :

$$
\Gamma_{1}\left(k_{0}\right)=\frac{1}{N_{0}} \mathbf{r}_{0}^{H} \mathbf{B}\left(\mathbf{B}^{H} \mathbf{B}+\mathbf{R}_{\mathbf{A}}\right)^{-1} \mathbf{B}^{H} \mathbf{r}_{0}
$$

The estimate of $k_{0}$ is then given by

$$
\hat{k}_{0}=\arg \max _{k_{0}}\left\{\Gamma_{1}\left(k_{0}\right)\right\} .
$$

A second approach to cope with the presence of the unknown data symbols in (7) is to totally neglect the contributions of the unknown data symbols. This means that we neglect $\mathbf{A}$ in (7) and (8). In that case the estimate of $\mathbf{h}$ given $k_{0}$ is given by

$$
\hat{\mathbf{h}}\left(k_{0}\right)=\left(\mathbf{B}^{H} \mathbf{B}\right)^{-1} \mathbf{B}^{H} \mathbf{r}_{0}
$$

and the estimate of $k_{0}$ is then given by

$$
\hat{k}_{0}=\arg \max _{k_{0}}\left\{\Gamma_{2}\left(k_{0}\right)\right\}
$$

with

$$
\Gamma_{2}\left(k_{0}\right)=\frac{1}{N_{0}} \mathbf{r}_{0}^{H} \mathbf{B}\left(\mathbf{B}^{H} \mathbf{B}\right)^{-1} \mathbf{B}^{H} \mathbf{r}_{0} .
$$

The maximization of both $\Gamma_{1}\left(k_{0}\right)$ and $\Gamma_{2}\left(k_{0}\right)$ can-not be performed analytically and therefore a one-dimensional search procedure is applied.

Although (10) or (13) can be used to estimate $\mathbf{h}$ after obtaining $\hat{k}_{0}$ from (12) or (14), we are not planning to use these estimates, because they perform poorly at high $E_{s} / N_{0}$. Neglecting the contributions from the data symbols in (8) and (9) results in an error floor in the MSE of $\mathbf{h}$ and in the resulting BER (see [3] and [4]). Actually, (10) and (13) are only used to obtain $\Gamma_{1}\left(k_{0}\right)$ and $\Gamma_{2}\left(k_{0}\right)$. In the literature, better channel estimators are available, e.g. [12] and [13], that perform better at high $E_{s} / N_{0}$ than the estimators (10) and (13).

The functions $\Gamma_{1}\left(k_{0}\right)$ and $\Gamma_{2}\left(k_{0}\right)$ have a similar structure. They both compute the correlation between the received signal and the pilot vector $\mathbf{b}$ at $\mathrm{L}$ successive time instants as be can be concluded from the matrix product $\mathbf{B}^{H} \mathbf{r}_{0}$ :

$$
\begin{aligned}
\left(\mathbf{B}^{H} \mathbf{r}_{0}\right)_{l} & =\sum_{k=0}^{N-1} r\left(k_{0}+l+k\right)\left(s_{p}(k)\right)^{*} \\
& +\sqrt{\frac{N}{N+\nu}}\left(\sum_{k=0}^{\nu-1} r\left(k_{0}+l+N+k\right)\left(b_{g}(k)\right)^{*}\right.
\end{aligned}
$$

where $l=0, \ldots, L-1$. Both the estimators (12) and (14) search the $\hat{k}_{0}$ that maximizes a function of the $L$ successive correlations between the received signal and the pilot vector. 


\section{Simulation Results}

In this section we examine the performance of the proposed time delay estimators by means of simulations. These estimators are compared to the time delay estimator for $\mathrm{CP}$ OFDM from [11]. We consider $N=1024$ carriers, a guard interval of length $\nu=74$ and $M-\nu$ pilot symbols transmitted on the carriers, for both KSP-OFDM and CP-OFDM ${ }^{2}$. The transmitted symbols consist of randomly generated QPSK symbols. Although we derived the estimator for $k_{0}$ under the assumption that only one OFDM block is transmitted, we simulate the transmission of consecutive OFDM blocks.

The performance of the estimators in a dispersive channel is illustrated in figures 3-5. The considered channel is a frequency selective Rayleigh fading channel consisting of $L=$ 50 channel taps with equal variance. For channel estimation, we consider the estimators from [13] and [14] for KSP-OFDM and $\mathrm{CP}-\mathrm{OFDM}$ respectively. Both algorithms obtain the least squares estimate of the channel impulse response, based on the FFT outputs on the pilot carrier positions of the received OFDM block. A CP-OFDM can compensate a time delay estimation error, provided that the estimated starting point is inside the interval [7] and the CP-OFDM system estimates the channel impulse response during the whole cyclic prefix. This means that the CP-OFDM system has to estimate $\nu+1$ channel coefficients although the original channel impulse response only consists of $L$ coefficients. To distinguish the two KSPOFDM estimators, the estimator from (12) which takes the unknown data symbols in to account, is labeled ' $K S P$-OFDM estimator 1', while the estimator from (14) which neglects the contributions from the unknown data symbols, is called 'KSP-OFDM estimator 2'.

In figures 3 and 4 a histogram of the estimation error $\hat{k}_{0}-k_{0}$ for the KSP-OFDM estimators and the CP-estimator respectively, is shown for $E_{s} / N_{0}=20 \mathrm{~dB}$ and 100 pilot carriers $(M=174)$. The probability that the KSP-OFDM estimators 1 and 2 find the true $k_{0}$ is more than $80 \%$ and $90 \%$ respectively. The estimation error $\left|\hat{k}_{0}-k_{0}\right|$ is smaller than or equal to 2 samples in more than $99 \%$ of all simulated cases for both KSP-OFDM estimators. The considered CP-OFDM estimator from [11] performs worse: the true $k_{0}$ is found in only $1 \%$ of all cases, but thanks to the presence of the cyclic prefix, the CP-OFDM system is able to reach the performance of a CP-OFDM system with perfect synchronization when the estimate $\hat{k}_{0}$ is in the interval $\left[k_{0}-\nu+L-1, k_{0}\right]=$ $\left[k_{0}-25, k_{0}\right]$. According to figure 4 , we see that in more $99 \%$ of the cases, the estimate $\hat{k}_{0}$ is in the desired interval. The CPOFDM time delay estimator exhibits a larger variance which means that the cyclic prefix must be long enough to avoid that the estimation error causes IBI.

Figure 5 shows the BER results for a dispersive Rayleigh fading channel. First of all, we observe that the curves

\footnotetext{
${ }^{2}$ By taking $N, \nu$ and the number $M-\nu$ of pilot carriers the same for both CP-OFDM and KSP-OFDM, we obtain the same data throughput and, assuming perfect synchronization and channel knowledge, essentially the same BER.
}
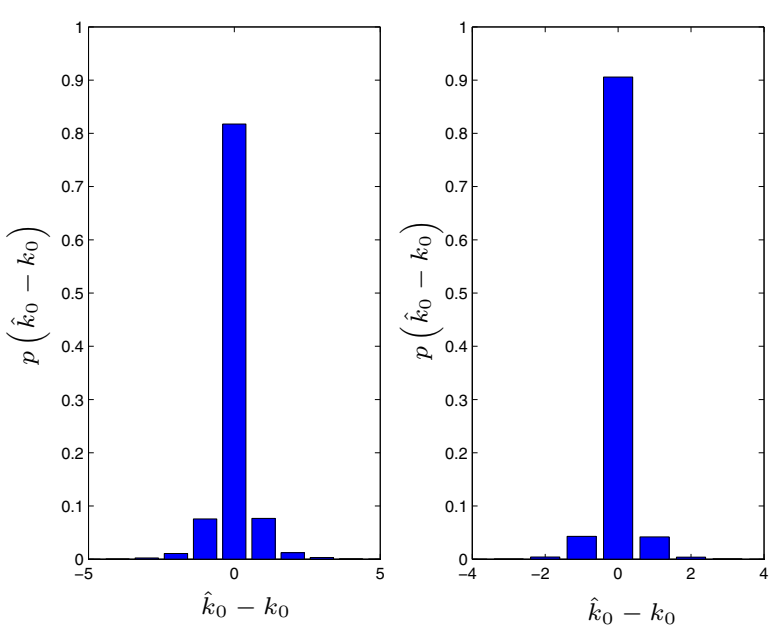

Fig. 3. Histogram of the time delay estimation error for the KSP-OFDM estimator 1 (left) and 2 (right), $E_{s} / N_{0}=20 \mathrm{~dB}, M=174$

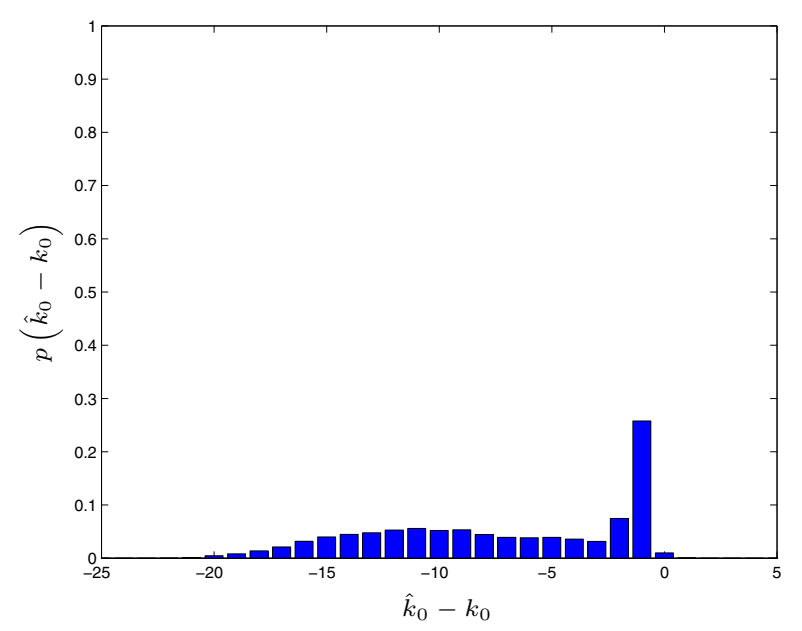

Fig. 4. Histogram of the time delay estimation error for the CP-OFDM estimator, $E_{s} / N_{0}=20 \mathrm{~dB}, M=174$

for both KSP-OFDM and CP-OFDM systems with perfect synchronization and perfect channel state information (CSI) are essentially the same. The BER results for perfect synchronization and estimated channel indicate that KSP-OFDM outperforms CP-OFDM. This is caused by the extra number of samples of the channel impulse response that have to be estimated in the CP-OFDM system. The dashed lines show the performance of the KSP-OFDM and the CP-OFDM system with time delay estimation and channel estimation. For the KSP-OFDM system, only the results for the second time delay estimator (14) are shown because it outperforms the first time delay estimator (12) as can be seen from figure 3 . For low to moderate $E_{s} / N_{0}$, both OFDM systems with time delay estimation are very close to the OFDM systems with perfect synchronization, so the KSP-OFDM system still outperforms the CP-OFDM system. For high $E_{s} / N_{0}$, we observe an error 


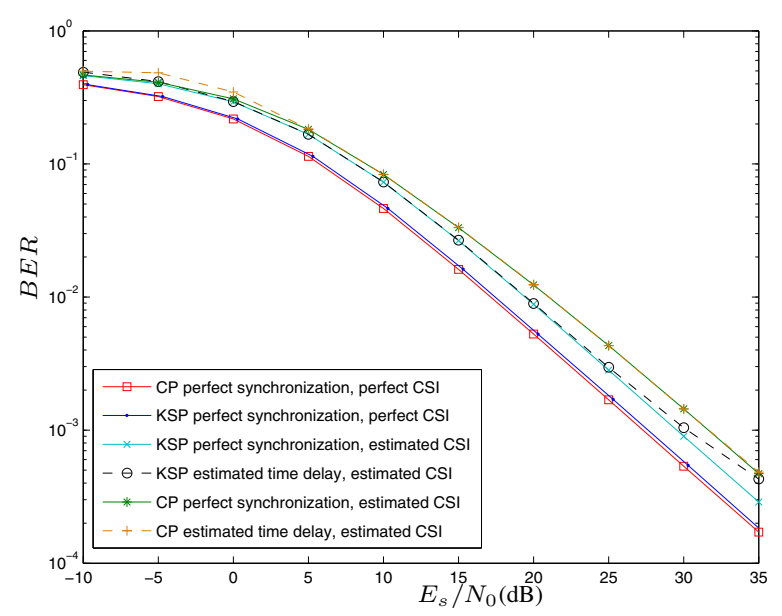

Fig. 5. BER results for a frequency selective channel, $L=50, N=1024$, $\nu=74, M=174$

floor for the KSP-OFDM system which is caused by the assumptions made for the derivation of the estimator, whereas no such floor occurs for CP-OFDM.

\section{CONCLUSION}

In this paper, two time delay estimators for KSP-OFDM in multipath fading channels are derived. These estimators exploit both the pilot symbols in the guard interval and the pilot symbols on the pilot carriers. The first estimator takes the unknown data symbols in to account while the second one totally neglects them. The performance of both proposed estimators is compared to the performance of the time delay estimator for CP-OFDM from [11]. As compared to CPOFDM, the time delay estimators for KSP-OFDM result in a considerably smaller variance on the estimate. For moderate $E_{s} / N_{0}$, KSP-OFDM (with estimation of time delay) gives rise to the smaller BER. The KSP-OFDM time delay estimator which totally neglects the contribution from the data symbols, yields the better results.

\section{APPENDIX}

In this appendix we compute $\mathbf{R}_{\mathbf{A}}$ which is the average of $\mathbf{A}^{H} \mathbf{A}$. Note that $\mathbf{A}^{H} \mathbf{A}$ is a Hermitian symmetric matrix, so it is sufficient to only consider the elements $(k, l)$ with $l \geq k$. The elements of $\mathbf{A}^{H} \mathbf{A}$ are given by

$$
\begin{array}{r}
\left(\mathbf{A}^{H} \mathbf{A}\right)_{k, l}=\sum_{m=0}^{N-1-(l-k)}\left(s_{d}(m+l-k)\right)^{*} s_{d}(m) \\
l \geq k, k=0, \ldots, L-1
\end{array}
$$

where $s_{d}(m)$ are the elements of the vector $\mathbf{s}_{d}$, defined in (3). Averaging those elements over the unknown data symbols yields for the elements of $\mathbf{R}_{\mathbf{A}}$

$$
\begin{array}{r}
\left(\mathbf{R}_{\mathbf{A}}\right)_{k, l}=(N-l+k) \frac{E_{s}}{N+\nu} \sum_{m=0}^{N-M+\nu-1} e^{-j 2 \pi \frac{n_{m}(l-k)}{N}} \\
l \geq k, k=0, \ldots, L-1
\end{array}
$$

\section{REFERENCES}

[1] J. A. C. Bingham. "Multicarrier modulation for data transmission, an idea whose time has come". IEEE Comm. Mag., 28(5):5-14, May 1990.

[2] B. Muquet, Z. Wang, G.B. Giannakis, M. de Courville and P. Duhamel. "Cyclic prefixing or zero padding for wireless multicarrier transmissions". IEEE Trans. Comm., 50(12):2136-2148, December 2002.

[3] O. Rousseaux, G. Leus and M. Moonen. "Estimation and Equalization of Doubly Selective Channels Using Known Symbol Padding". IEEE Trans. Signal Proc., 54(3):pp. 979-990, March 2006.

[4] H. Steendam and M. Moeneclaey. "Different Guard Interval Techniques for OFDM: Performance Comparison". In Proc. from 6th International Workshop on Multi-Carrier Spread Spectrum (MC-SS'07), pages pp. 1124, Herrsching, Germany, May 2007.

[5] H. Minn, V. K. Bhargava and B. Letaief. "A Robust Timing and Frequency Synchronization for OFDM Systems". IEEE Trans. Wireless Comm., 2(4):pp. 822-839, July 2003.

[6] T. Keller, L. Piazzo, P. Mandarini and L. Hanzo. "Orthogonal Frequency Division Multiplex Synchronization Techniques for Frequency-Selective Fading Channels". IEEE Journal on Selected Areas in Comm., 19(6):pp. 999-1008, June 2001.

[7] Y. Mostofi and D. C. Cox. "Mathematical Analysis of the Impact of Timing Synchronization Errors on the Performance of an OFDM System". IEEE Trans. Comm., 54(2):pp. 226-230, February 2006.

[8] D. Landström, S.K. Wilson, J.-J. van de Beek; P. Ödling and P.O. Börjesson. "Symbol Time Offset Estimation in Coherent OFDM Systems". IEEE Trans. Comm., 50(4):pp. 545-549, April 2002.

[9] T. Schmidl and D.C. Cox. "Robust Frequency and Timing Synchronization for OFDM". IEEE Trans. Comm., 45(12):1613-1621, December 1997.

[10] J.-J. van de Beek, P. O. Börjesson, M.-L. Boucheret, D. Landström, J. M. Arenas, P. Ödling, S. K. Wilson. "Three non-pilot based time- and frequency estimators for OFDM". Elsevier Signal Processing, 80:pp. 1321-1334, 2000.

[11] B. Yang, K. B. Letaief, R. S. Cheng and Z. Cao. "Timing Recovery for OFDM Transmission". IEEE Journal on Selected Areas in Comm., 18(11):pp. 2278-2291, November 2000.

[12] H. Steendam, M. Moeneclaey and H. Bruneel. "An ML-Based Estimate and the Cramer-Rao Bound for Data-Aided Channel Estimation in KSP-OFDM". EURASIP Journal on Wireless Communications and Networking, special issue on Multicarrier Systems, 2008. Article ID 186809, 9 pages, 2008. doi:10.1155/2008/186809.

[13] D. Van Welden, H. Steendam and M. Moeneclaey. "FrequencyDomain Data-Aided Channel Estimation for KSP-OFDM". In Proc. from 10th International Symposium on Spread Spectrum Techniques and Applications (ISSSTA'08), Bologna, Italy, August 2008.

[14] J.-J. van de Beek, O. Edfors, M. Sandell, S. K. Wilson and P. O. Börjesson. "On Channel Estimation in OFDM Systems". In Proc. Vehicular Technology Conference (VTC '95), Chicago, USA, September 1995. 